\title{
Muillerianosis of the Urinary Bladder: A Rare Condition Which Mimics Neoplasia
}

\author{
Somshukla Ghosh ${ }^{1}$, Fikret Onol ${ }^{2}$, Jonathan Vasquez ${ }^{3}$, Jignesh Parikh ${ }^{3}$ \\ 1. Internal Medicine, University of Central Florida-HCA Healthcare Graduate Medical Education, Greater Orlando, USA \\ 2. Urology, Orlando Veterans Affairs Medical Center, Orlando, USA 3. Pathology, Orlando Veterans Affairs Medical \\ Center, Orlando, USA
}

Corresponding author: Somshukla Ghosh, somshukla.ghosh@ucf.edu

\begin{abstract}
Müllerianosis of the urinary bladder is an extremely rare, benign condition defined by the presence of at least two of the three müllerian-derived components (endosalpinx, endometrium, and endocervix) in the lamina propria and muscularis propria of the urinary bladder. It frequently mimics neoplastic condition, either malignant or benign. Here, we present a case of cystic müllerianosis of urinary bladder, which was clinically thought to be a urinary bladder neoplasm.
\end{abstract}

Categories: Urology

Keywords: pseudoneoplastic bladder lesion, urinary bladder malignancy, hematuria, pelvic pain, müllerianosis of urinary bladder

\section{Introduction}

Müllerianosis of the urinary bladder is a benign condition characterized histopathologically by the presence of at least two of the three müllerian-derived components (endosalpinx, endometrium, and endocervix) in the lamina propria and muscularis propria of the urinary bladder [1]. It is an extremely rare condition, mostly affecting women in the child-bearing age group [2]. Symptoms include abnormal uterine bleeding, hematuria, dysuria, pelvic pain, and suprapubic pain. Radiologically, müllerianosis can be seen as a polypoid mass by ultrasound, computed tomogramy (CT), or magnetic resonance imaging (MRI). However, a preferred diagnostic imaging modality has not been identified because of the small number of reported patients with this condition. Differential diagnosis encompasses neoplastic conditions including invasive cancer of the genitourinary tract, and hence, accurate diagnosis is crucial for appropriate treatment. The potential for urinary bladder müllerianosis to transform into malignant neoplasia and recommended treatment modalities are also unclear.

Review began 05/04/2021 Review ended 05/18/2021 Published 05/21/2021

(๑) Copyright 2021

Ghosh et al. This is an open access article distributed under the terms of the Creative Commons Attribution License CC-BY 4.0., which permits unrestricted use, distribution, and reproduction in any medium, provided the original author and source are credited.

\section{Case Presentation}

A 31-year-old nulliparous woman presented with the complaints of menorrhagia and pelvic pain for seven months. She also complained of pain during micturition and increased urinary frequency during her menstrual cycles for the same duration. Contrast-enhanced CT scan of the abdomen and pelvis showed multiple uterine fibroids with both subserosal and submucosal components. To relieve her bulk symptoms, she underwent laparoscopic total hysterectomy with bilateral salpingectomy. During the laparoscopic surgery, a 2-3 cm nodule was visualized above the urinary bladder trigone. The nodule was hard and did not extend through the bladder serosa. Of note, the CT scan prior to the surgery did not show this lesion.

For further evaluation, the patient underwent an elective cystoscopy which revealed a nodular lesion with calcification on the posterior wall of the urinary bladder, which was highly suspicious for a neoplasm. The patient underwent a transurethral resection of this urinary bladder nodule. Histopathological examination showed a cyst within the urinary bladder wall, lined by tubal-type epithelium and endometriallike pericystic stroma (Figure 1). CD10 staining (Figure 2) was focally positive within pericysticstromal tissue, and estrogen receptor (ER) (Figure 3) and progesterone receptor (PR) (Figure 4) immunostains were positive within the cyst lining cells. The morphology and immunohistochemical stains supported the diagnosis of cystic müllerianosis. Her urinary symptoms resolved after its resection, and she did not show any signs of recurrence during a follow-up of 12 months. 


\section{Cureus}

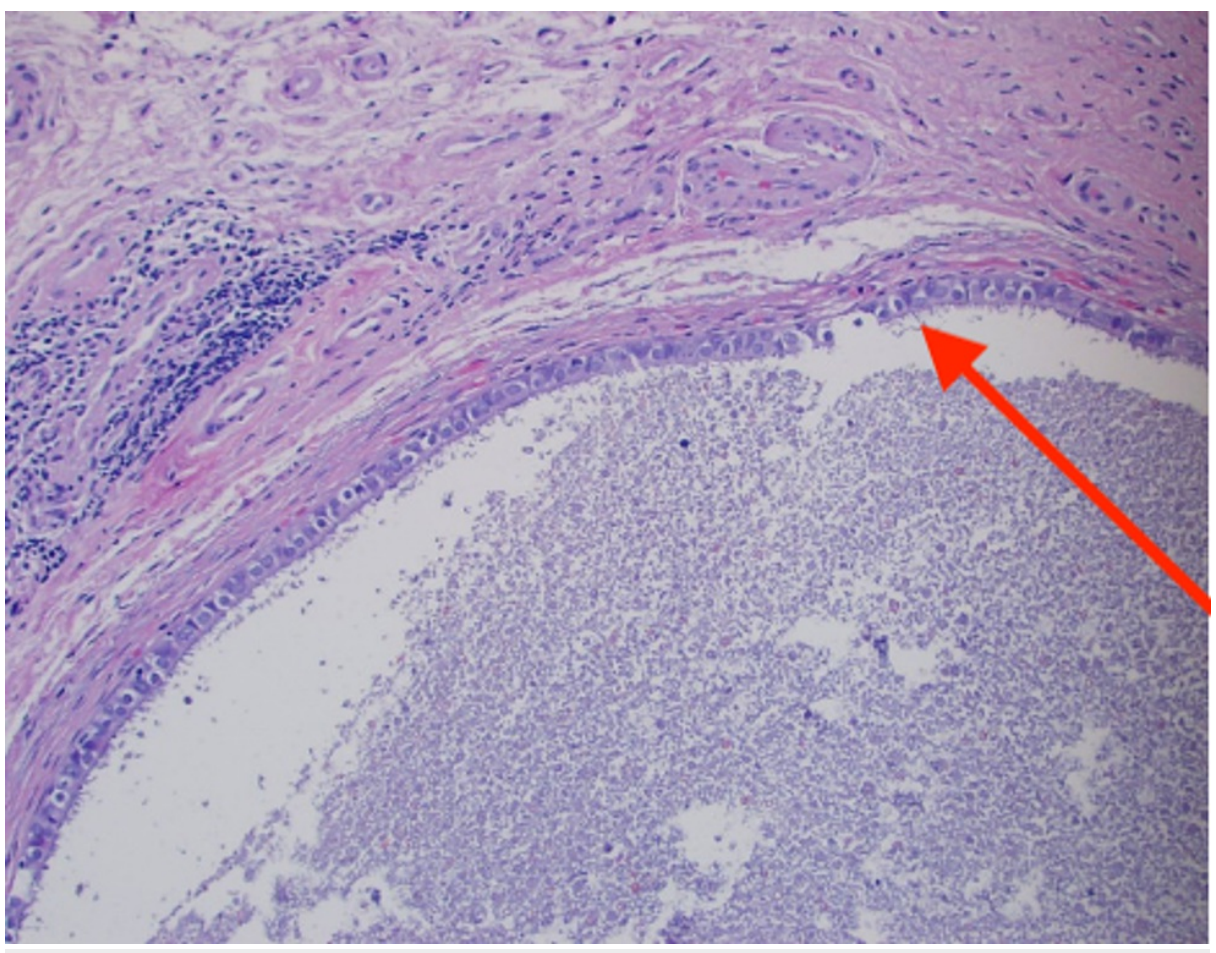

FIGURE 1: Hematoxylin and eosin-stained section showing ciliated epithelium-lined cyst.

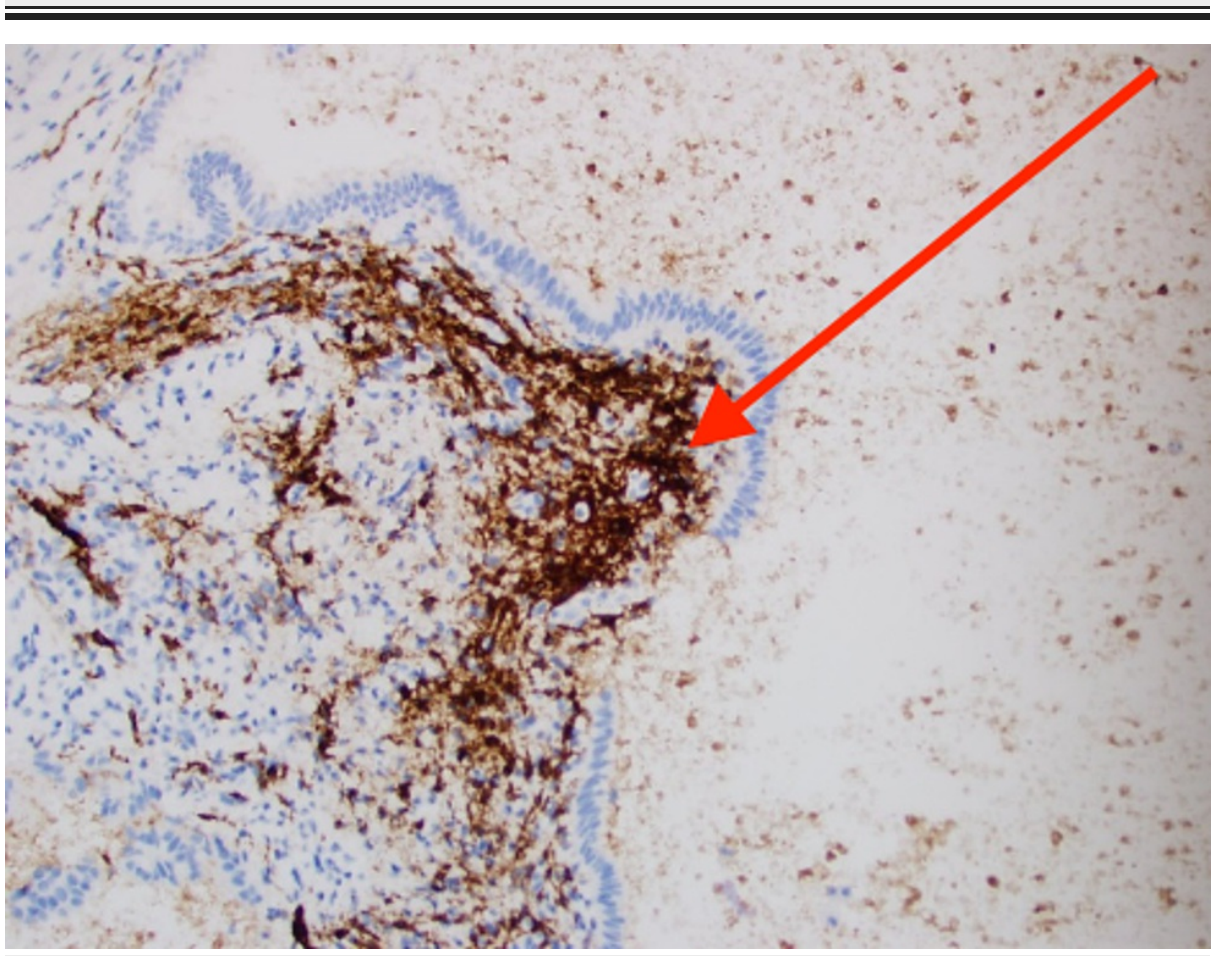

FIGURE 2: Pericystic stromal tissue focally positive for CD10 immunostain. 


\section{Cureus}

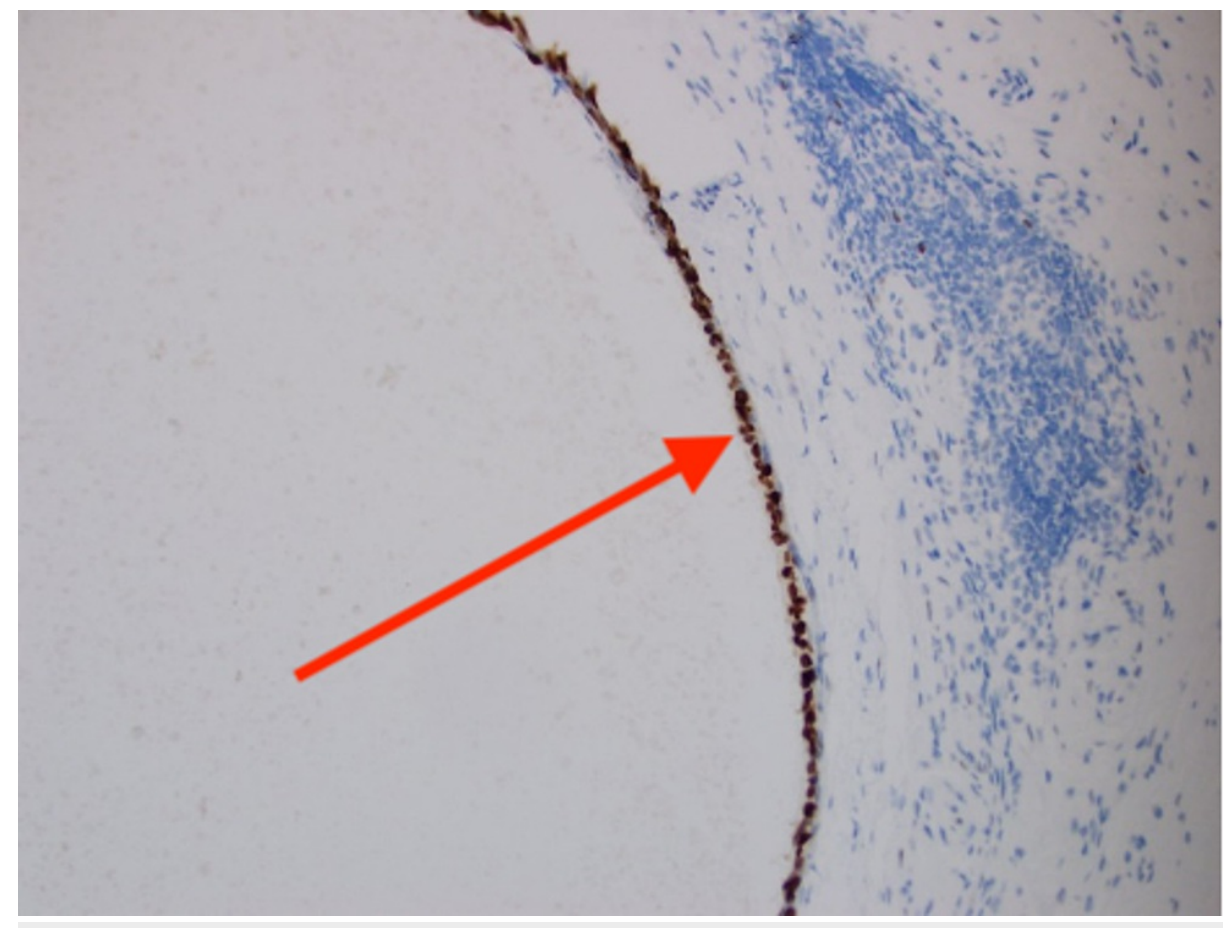

FIGURE 3: Cyst lining cells positive for immunostains for ER.

ER: estrogen receptor

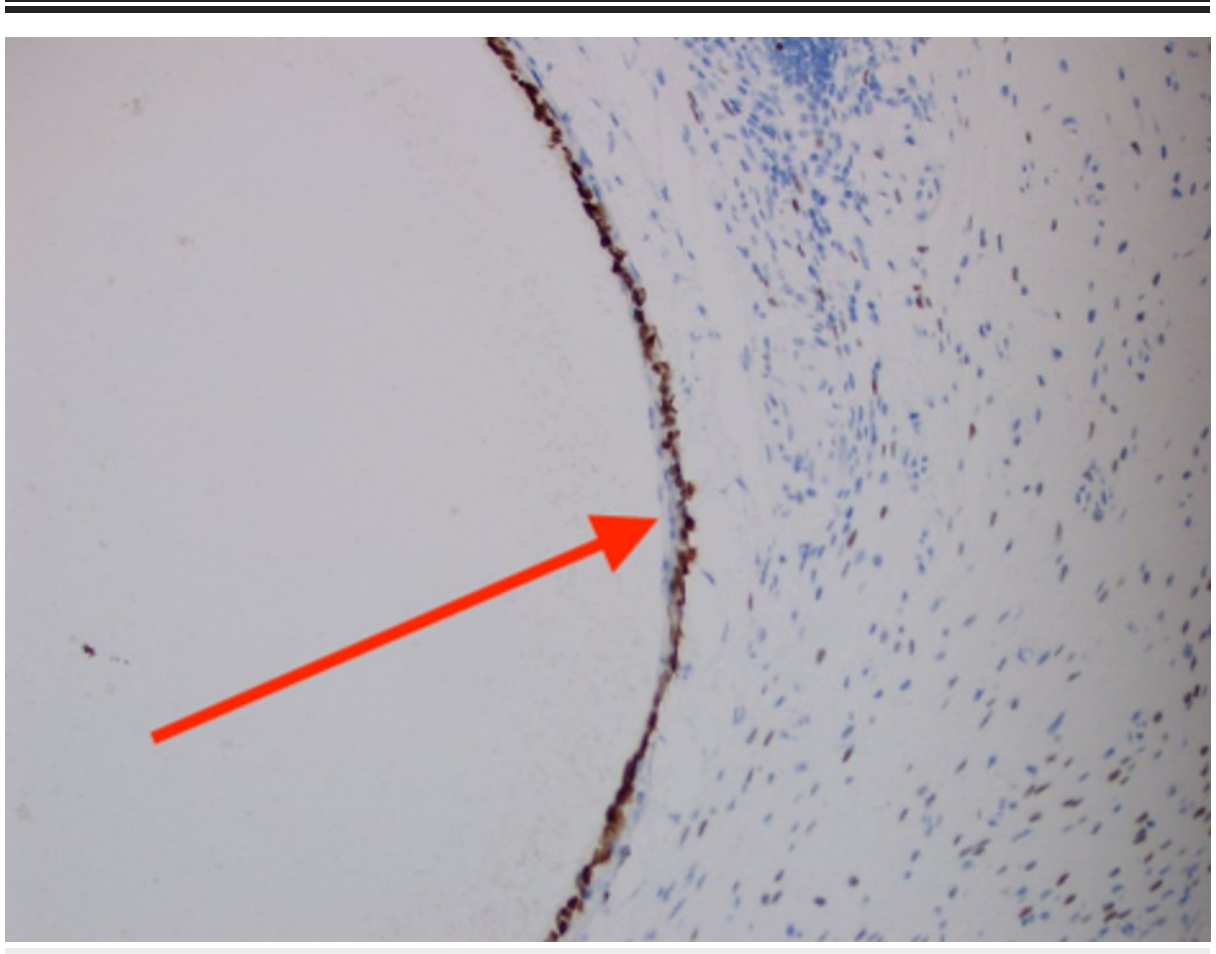

FIGURE 4: Cyst lining cells positive for immunostains for PR.

PR: progesterone receptor

\section{Discussion}

Müllerianosis of the urinary tract is an extremely rare condition with less than 40 cases reported to date [214]. It is a benign condition and the most common sites reported are the trigone or posterior wall of the urinary bladder, or the lower ureter or the ureterovesical junction. So far, all the cases reported have been in 
women, mostly in the child-bearing age group. There have been only three reported cases so far in postmenopausal women $[3,13,15]$. This condition is characterized histopathologically by the presence of at least two of the three müllerian-derived components (endosalpinx, endometrium, and endocervix) in the lamina propria and muscularis propria of the urinary bladder [1]. However, there are also cases reported where the bladder lesion, on histopathology, has shown tubal-type epithelium only [16]. To our knowledge, only five such cases have been documented in the English language literature [16-19], and this entity is called endosalpingosis.

Symptoms include abnormal uterine bleeding, hematuria, dysuria, pelvic pain, and suprapubic pain, which may or may not be associated with menstruation. Patients with ureteric lesions often had symptoms suggestive of ureteric obstruction or hematuria. The symptoms of our patient were associated with menstruation.

Both implantative and metaplastic origins have been suggested as pathogenesis of these lesions $[3,18]$. In nine of the cases reported previously, the patients had prior history of pelvic surgery or caesarian section, thus supporting the implantative pathology. However, our patient was nulliparous and did not have any history of pelvic surgery; hence, the likelihood of metaplastic origin is higher.

Ultrasound, MRI, or urinary cytology showing epithelial aggregates with papillary configurations have been useful for the diagnosis. Radiologically, müllerianosis can be seen as a polypoid mass on ultrasound, CT scan, or MRI. A preferred diagnostic imaging modality has not been identified because of such a small number of patients that have been reported with this condition. Interestingly, CT scan of the abdomen and pelvis did not reveal any urinary bladder nodule in our patient, and it was only seen on cystoscopy. There have been two cases reported in which the diagnosis was suggested by urine cytopathology which showed endometrium-like cells [12].

Differential diagnosis of müllerianosis of the bladder includes cystitis glandularis, cystitis cystica, bladder adenocarcinoma, carcinoma of the urachus, and neoplastic lesions, including cervical cancer invading into the bladder wall. Definitive diagnosis is by histopathologic evaluation after cystoscopic removal of the lesion.

Treatment of müllerianosis is controversial, and modalities include pharmacotherapy with gonadotropinreleasing hormone $(\mathrm{GnRH})$ agonist or with surgical resection of the lesion. Two cases have been reported where treatment with GnRH resulted in resolution of symptoms; however, the size of the lesion continued to persist [3,11]. Because of the concern for its potential malignant transformation [7], resection seems to be the preferred treatment.

Despite the benign nature of bladder müllerianosis, some reports have described malignant transformation of the müllerianosis into bladder endometroid carcinoma [7]. Due to very limited number of known cases, the potential for bladder müllerianosis to transform into malignant neoplasia is unknown. However, patients should be followed up with cystoscopy even after successful surgical removal of the lesion. They should also be monitored for recurrence of symptoms. There is no recommended time interval for repeat cystoscopies due to the very small number of cases. Our patient has been doing well with complete resolution of all her symptoms at one-year follow-up after the removal of the mass. She is yet to have a repeat cystoscopy.

In summary, müllerianosis of the urinary bladder closely mimics a urinary bladder neoplastic lesion in terms of clinical presentation, cytology, and cystoscopic findings [11,19]. Accurate diagnosis is crucial as correct diagnosis will prevent the patient from undergoing radical cystectomy which is the standard treatment for invasive urinary bladder malignancy.

\section{Conclusions}

Müllerianosis is a rare condition that can be considered to be a pseudoneoplastic bladder lesion potentially responsible for a false diagnosis of malignancy. Due to its rarity, definitive guidelines for management are lacking. Recommended imaging modality, probability of malignant transformation, and time interval for surveillance are few of the many areas that need further exploration. We report this case to add to the general knowledge about müllerianosis.

\section{Additional Information \\ Disclosures}

Human subjects: Consent was obtained or waived by all participants in this study. Conflicts of interest: In compliance with the ICMJE uniform disclosure form, all authors declare the following: Payment/services info: All authors have declared that no financial support was received from any organization for the submitted work. Financial relationships: All authors have declared that they have no financial relationships at present or within the previous three years with any organizations that might have an interest in the submitted work. Other relationships: All authors have declared that there are no other relationships or activities that could appear to have influenced the submitted work. 


\section{References}

1. Young RH, Clement PB: Müllerianosis of the urinary bladder. Mod Pathol. 1996, 9:731-7.

2. Habiba M, Brosens I, Benagiano G: Müllerianosis, endocervicosis, and endosalpingiosis of the urinary tract: a literature review. Reprod Sci. 2018, 25:1607-18. 10.1177/1933719118773441

3. Donné C, Vidal M, Buttin X, Becerra P, Carvia R, Zuluaga A, Nogales FF: Müllerianosis of the urinary bladder: clinical and immunohistochemical findings. Histopathology. 1998, 33:290-2. 10.1046/j.13652559.1998.0482f.x

4. Jiménez-Heffernan JA, Sánchez-Piedra D, Bernaldo de Quiros L, Martínez V: Endosalpingiosis (müllerianosis) of the bladder: a potential source of error in urinary cytology. Cytopathology. 2000, 11:34853. 10.1046/j.1365-2303.2000.00262.x

5. Margulis V, Lemack GE, Molberg K, Saboorian MH: Bladder "müllerianosis" in a woman with lower urinary tract symptoms and hematuria. J Urol. 2001, 165:1996-7. 10.1097/00005392-200106000-00043

6. Islam S, Tumman JJ, McMahon RF, Payne SR: Müllerianosis of the urinary bladder. BJU Int. 2003, 92 Suppl 3:e23. 10.1111/j.1464-410x.2003.04036.x

7. Garavan F, Grainger R, Jeffers M: Endometrioid carcinoma of the urinary bladder complicating vesical Mullerianosis: a case report and review of the literature. Virchows Arch. 2004, 444:587-9. 10.1007/s00428004-1010-8

8. Koren J, Mensikova J, Mukensnabl P, Zamecnik M: Mullerianosis of the urinary bladder: report of a case with suggested metaplastic origin. Virchows Arch. 2006, 449:268-71. 10.1007/s00428-006-0153-1

9. Olivia Vella JE, Nair N, Ferryman SR, Athavale R, Latthe P, Hirschowitz L: Müllerianosis of the urinary bladder. Int J Surg Pathol. 2011, 19:548-51. 10.1177/1066896911409578

10. Ogah K, Hartis R, Hilton P: Mullerianosis involving the urinary bladder. Int Urogynecol J. 2012, 23:123-5. 10.1007/s00192-011-1455-7

11. Kudva R, Hegde P: Mullerianosis of the urinary bladder. Indian J Urol. 2012, 28:206-7. 10.4103/09701591.98469

12. Guan H, Rosenthal DL, Erozan YS: Mullerianosis of the urinary bladder: report of a case with diagnosis suggested in urine cytology and review of literature. Diagn Cytopathol. 2012, 40:997-1001. 10.1002/dc. 21693

13. McSorley S, Kochman A, DeSouza J: Grand rounds: müllerianosis of the urinary bladder in a postmenopausal woman. Urology. 2013, 81:488-90. 10.1016/j.urology.2012.11.013

14. Salada RB, Yong D, Ho CSB, Chong YL: Müllerianosis: a case report. J Endourol Case Rep. 2019, 5:124-7. 10.1089/cren.2019.0036

15. Heretis J, Stamatiou K, Papadimitriou V, Giannikaki E, Stathopoulos E, Sofras F: Endocervicosis of the bladder: report of a case and review of the current literature. Int J Clin Exp Pathol. 2009, 2:91-4.

16. Edmondson JD, Vogeley KJ, Howell JD, Koontz WW, Koo HP, Amaker B: Endosalpingiosis of bladder. J Urol. 2002, 167:1401-2. 10.1016/S0022-5347(05)65318-9

17. Smith C, Sabet L, Izawa JI: Management of endosalpingiosis of urinary bladder . Urology. 2004, 64:1031. 10.1016/j.urology.2004.05.041

18. Maniar KP, Kalir TL, Palese MA, Unger PD: Endosalpingiosis of the urinary bladder: a case of probable implantative origin with characterization of benign Fallopian tube immunohistochemistry. Int J Surg Pathol. 2010, 18:381-3. 10.1177/1066896909332730

19. Mincik I, Mytnik M, Straka L, Breza J Jr, Vilcha I: Inflammatory pseudotumour of urinary bladder - a rare cause of massive macroscopic haematuria. Bratisl Lek Listy. 2013, 114:495-7. 10.4149/bll_2013_103 